Meta

Journal des traducteurs

Translators' Journal

\title{
Des zones d'indécidabilité dans la traduction automatique et dans la traduction humaine
}

\section{Silvia Kadiu}

Volume 61, numéro 1, mai 2016

Des zones d'incertitudes en traduction

URI : https://id.erudit.org/iderudit/1036990ar

DOI : https://doi.org/10.7202/1036990ar

Aller au sommaire du numéro

Éditeur(s)

Les Presses de l’Université de Montréal

ISSN

0026-0452 (imprimé)

1492-1421 (numérique)

Découvrir la revue

Citer cet article

Kadiu, S. (2016). Des zones d'indécidabilité dans la traduction automatique et dans la traduction humaine. Meta, 61(1), 204-220.

https://doi.org/10.7202/1036990ar
Résumé de l'article

Cet article examine le concept d'indécidabilité de Jacques Derrida dans la traduction automatique et dans la traduction humaine. Définie comme la condition même de toute possibilité d'action et de décision (Caputo 1997 : 137), l'indécidabilité est la composante structurelle de toute décision. S'appuyant sur le concept de Derrida pour penser la complexité des processus décisionnels à l'oeuvre dans l'acte traductif, cet article explore les zones d'indécidabilité dans les traductions d'un extrait de Traduire au XXI ${ }^{\mathrm{e}}$ siècle d'Henri Meschonnic (2008). Délibérément choisi pour sa résonance directe avec la notion d'indécidabilité, le commentaire de Meschonnic soutient que, mis à part les traductions automatiques, aucune pratique de la traduction n'est possible sans prise de décision réflexive (Meschonnic 2008 : 60). Les observations traitées dans cet article remettent en question l'opposition homme-machine en traduction en montrant que la programmabilité et l'indécidabilité sont des caractéristiques communes à la traduction automatique et à la traduction humaine. Cette étude semble ainsi indiquer que, comme condition préalable à toute prise de décision éthique, l'incertitude doit être développée au sein même des systèmes de traduction automatique et qu'une formation approfondie des traducteurs aux technologies de la traduction est indispensable pour un usage responsable de la traduction automatique.
Ce document est protégé par la loi sur le droit d'auteur. L'utilisation des services d’Érudit (y compris la reproduction) est assujettie à sa politique d'utilisation que vous pouvez consulter en ligne.

https://apropos.erudit.org/fr/usagers/politique-dutilisation/ 


\title{
Des zones d'indécidabilité dans la traduction automatique et dans la traduction humaine
}

\author{
SILVIA KA DIU \\ University College London, Londres, Royaume-Uni \\ silvia.kadiu.11@ucl.ac.uk
}

\begin{abstract}
RÉSUMÉ
Cet article examine le concept d'indécidabilité de Jacques Derrida dans la traduction automatique et dans la traduction humaine. Définie comme la condition même de toute possibilité d'action et de décision (Caputo 1997: 137), l'indécidabilité est la composante structurelle de toute décision. S'appuyant sur le concept de Derrida pour penser la complexité des processus décisionnels à l'œuvre dans l'acte traductif, cet article explore les zones d'indécidabilité dans les traductions d'un extrait de Traduire au Xxl siècle d'Henri Meschonnic (2008). Délibérément choisi pour sa résonance directe avec la notion d'indécidabilité, le commentaire de Meschonnic soutient que, mis à part les traductions automatiques, aucune pratique de la traduction n'est possible sans prise de décision réflexive (Meschonnic 2008: 60). Les observations traitées dans cet article remettent en question l'opposition homme-machine en traduction en montrant que la programmabilité et l'indécidabilité sont des caractéristiques communes à la traduction automatique et à la traduction humaine. Cette étude semble ainsi indiquer que, comme condition préalable à toute prise de décision éthique, l'incertitude doit être développée au sein même des systèmes de traduction automatique et qu'une formation approfondie des traducteurs aux technologies de la traduction est indispensable pour un usage responsable de la traduction automatique.
\end{abstract}

\section{ABSTRACT}

This article examines Jacques Derrida's concept of undecidability in machine and human translation. Defined as the very condition of possibility of acting and deciding (Caputo 1997: 137), undecidability is an essential component of decision-making. Using Derrida's concept as a starting point for thinking the intricacies of translation decision processes, this article explores manifestations of undecidability in the translations of an extract from Henri Meschonnic's Traduire au XXIe siècle (2008). Deliberately selected because of its direct resonance with the notion of undecidability, Meschonnic's comment claims that, except for automated translations, no practice of translation is possible without reflexive decision-making (Meschonnic 2008: 60). The observations discussed in this article challenge clear-cut distinctions between human and automated translations by showing that programmability and undecidability are features shared by both humans and machines in translation. As such, this study suggests that uncertainty, as a pre-condition of ethical decision-making, must be fostered within mechanical translations themselves, and that in-depth training in translation technology is necessary for a responsible use of machine translation.

\section{MOTS CLÉS/KEYWORDS}

indécidabilité, traduction automatique, réflexivité, traductologie, traductique undecidability, machine translation, reflexivity, translation studies, CAT 
Ce qui me gêne chez certains scientistes [...], c'est que leurs modèles mécaniques ne sont pas toujours à la mesure de l'hypercomplexité des machines, réelles ou virtuelles, produites par les hommes (et dont témoignent, par exemple, toutes les apories ou les «impossibles» qui occupent la « déconstruction» là même où elle met à l'épreuve, dans le langage, les plus puissantes machines à formaliser; non pas pour disqualifier la «machine» en général, bien au contraire, mais pour "penser» autrement l'évènement et l'historicité). À mon avis, la pensée la plus «libre» est celle qui transige tout le temps avec des effets de machine.

(Derrida 2001: 85)

\section{Introduction}

Le développement rapide de la traductologie ces trente dernières années a vu naître un nombre croissant de théories s'efforçant de rendre compte de l'activité du traduire à la fois dans sa spécificité (par comparaison avec d'autres formes d'écriture) et pardelà sa diversité (dans des champs aussi variés que la littérature, l'audiovisuel ou le juridique). Or si l'objectif premier d'une théorie est d'établir des «lois» concernant son objet d'étude (Compact Oxford English Dictionary 1971, cité dans Tymoczko 2013: 1), la multiplication des modèles traductifs qui a accompagné le développement de la traductologie ces dernières années semble au contraire avoir amplifié nos incertitudes à l'égard de ce qui constitue une «bonne» traduction. Faut-il traduire littéralement, en indiquant l'étrangeté du texte source, comme le préconisent chacun à leur façon Antoine Berman (1984) et Lawrence Venuti (1995), ou bien adopter l'approche phénoménologique de Clive Scott qui, dans Translating the Perception of Text (2012), présente la traduction comme l'expression d'une activité de lecture sensorielle et subjective? Doit-on résister à l'impulsion ethnocentrique de l'acte traductif en refusant parfois de traduire, comme semble l'indiquer Emily Apter dans Against World Literature: On the Politics of Untranslatability (2013)? Ou bien adapter son approche en fonction de l'objectif du texte traduit dans la culture cible, comme le prônent les penseurs de la théorie du Skopos (Reiss et Vermeer 1984/2014) ? Une chose est sûre, le débat entre sourciers et ciblistes reste de vigueur (Ladmiral 2014) et l'incertitude ambiante concernant la bonne manière de traduire n'a sans doute jamais été aussi palpable (Pym 2010). Cette incertitude semble prévaloir particulièrement dans les domaines poétique et littéraire, par opposition à la traduction technique ou automatique. Berman distingue ainsi la traductologie (l'étude de la traduction centrée sur la linguistique, la littérature comparée et la poétique) de la traductique, «dernièrenée des disciplines qui, dans le sillage de l'informatique, de la productique, etc., veulent, » selon lui, "annexer les "processus de traduction" à leurs systèmes de computation» (Berman 1999: 17).

C'est dans le cadre de cette opposition entre poétique et automation qu'Henri Meschonnic propose de définir la traduction comme une activité intrinsèquement réflexive. Dans Traduire au XXI siècle (2008), il soutient:

On peut donc considérer [...] que le problème majeur de la traduction est sa théorie du langage. Ce qui est bien d'emblée impliquer deux choses: l'inséparabilité entre ce que l'on appelle une théorie et ce qu'on appelle une pratique, c'est-à-dire qu'une pratique n'est pas une pratique si elle n'est pas réflexive ou réfléchie, ce n'est qu'un ânonnement de recettes apprises, et si elle est cette réflexivité, cette pratique implique nécessairement 
une théorie d'ensemble du langage; et réciproquement une théorie de la traduction qui ne serait pas la réflexion d'une pratique ne serait que de la linguistique de la langue appliquée sur du discours, c’est-à-dire de la non-pensée. (Meschonnic 2008:60)

Théorie et pratique ne peuvent être dissociées l'une de l'autre en traduction, affirme Meschonnic, car, sauf lorsque celle-ci est mécanique, toute pratique traductive implique un processus de réflexion, une prise de décision qui manifeste en retour son rapport à la langue et au langage. La pratique à laquelle Meschonnic fait allusion ici, c'est la traduction automatique, une traduction opérée intégralement ou en partie par le biais d'un programme informatique (Shuttleworth et Cowie 2007: 99). L'objectif de Meschonnic est de dénoncer les représentations du langage fondées sur le discontinu signifiant-signifié du signe linguistique, auxquelles a contribué selon lui l'essor de la traduction machine. "Les tentatives de traduction automatique dès la fin de la Seconde Guerre mondiale, » explique-t-il, «ont eu un effet sur le développement d'une linguistique de la traduction [comme] conceptualisation de la langue, dans les termes dualistes du signe» (Meschonnic 2008: 59). Pour Meschonnic, le discontinu signifiant-signifié caractéristique du fonctionnement de la traduction automatique est un obstacle majeur à l'avènement d'une pratique traductive fondée sur la continuité du rythme et la poétique. En ce sens, sa pensée établit une opposition hiérarchique entre d'une part, la traduction mécanique, automatique, irréfléchie, qui reposerait sur «un ânonnement de recettes apprises", et d'autre part, une traduction humaine créative et réflexive, qui «implique[rait] nécessairement une théorie d'ensemble du langage».

Cette distinction entre mécanique et poétique mérite toutefois plus ample réflexion. Car, si toute pratique traductive implique nécessairement un processus décisionnel réflexif, soit la traduction automatique ne devrait pas être considérée comme une pratique en premier lieu, soit l'idée que la réflexivité est inhérente à la traduction ne s'applique en réalité qu'à certains types de traduction. La présente étude explore la question de la réflexivité décisionnelle à l'œuvre dans l'acte traductif à travers une analyse comparée de plusieurs traductions automatiques du susmentionné extrait de Traduire au XXI siècle. Cette démarche, délibérément réflexive, est inspirée de celle de Jacques Derrida dans «Des Tours de Babel» (1985), texte dans lequel ce dernier décide d'entreprendre une traduction de l'illustre essai de Walter Benjamin «La tâche du traducteur» (1923/2000) pour tenter de penser la spécificité de l'expérience traductive:

Cet exemple singulier [le mot Babel], à la fois archétypique et allégorique, pourrait introduire à tous les problèmes dits théoriques de la traduction. Mais aucune théorisation, dès lors qu'elle se produit dans une langue, ne pourra dominer la performance babélienne. C'est une des raisons pour lesquelles je préfère ici, au lieu d'en traiter sur le mode théorique, tenter de traduire à ma manière la traduction d'un autre texte sur la traduction. (Derrida 1985: 219)

Pour Derrida, l'intraduisibilité du mot «Babel», résultant à la fois de son statut de nom propre et de son inéluctable polysémie (puisqu'il signifie simultanément «Dieu», "père» et "confusion»), soulève des incertitudes intrinsèques à la traduction dont seule une démarche elle-même traduisante peut rendre compte.

L'incertitude à laquelle les traducteurs doivent faire face lors du processus traductif est étroitement liée à la notion derridienne d'indécidabilité qui, contrairement à ce que l'on pourrait penser, ne fait pas référence à une certaine hésitation ou à 
l'incapacité de choisir, mais bien au contraire à la condition même de toute possibilité d'action et de décision. "Il ne faut pas confondre l'indécidable avec l'indécision", précise Derrida. L'indécidable «n'est pas un pathos, c'est une condition structurelle» (Derrida 1990: 210). C'est ce qui résiste au calcul; ce qui demeure imprévisible; ce qui dépasse le machinal. Tâchant de pallier la confusion qui entoure le concept d'indécidabilité, Caputo explique:

Undecidability is taken, or mistaken, to mean a pathetic state of apathy, the inability to act, paralyzed by the play of signifiers that dance before our eyes, like a deer caught in a headlight. But rather than an inability to act, undecidability is the condition of possibility of acting and deciding. For whenever a decision is really a decision, whenever it is more than a programmable, deducible, calculable, computable result of a logarithm, that is because it has passed through the 'ordeal of undecidability.' One way to keep this straight is to see that the opposite of 'undecidability' is not 'decisiveness' but programmability, calculability, computerizability, or formalizability. Decisionmaking, judgment, on the other hand, positively depends upon undecidability, which gives us something to decide. (Caputo 1997: 137)

Pour Derrida, toute décision est structurée par l'expérience de l'indécidable car « [u]ne décision qui serait déterminée par des causes programmables et calculables ne serait pas une décision - ce ne serait qu'un effet». Autrement dit, pour qu'une décision "advienne», "il faut qu'elle ne soit déterminée ni par un calcul, ni même par la limite du calculable» (Derrida 1990: 210). En ce sens, une décision n'est véritablement une décision que si elle résulte d'une réflexion, d'une incertitude qui dépasse l'ordre du programmable et du calculable, que si elle n'est pas l'application d'une règle ou d'une loi.

S'appuyant principalement sur le concept d'indécidabilité auquel le commentaire de Meschonnic fait indirectement écho, cet article propose de soumettre le texte du théoricien de Traduire au XXI siècle à l'épreuve de sa propre théorie afin d'explorer les implications concrètes d'une traductologie opposant le mécanique et le réflexif. Une telle opposition est-elle tenable en pratique? Que signifie-t-elle concrètement? Et quelles en sont les conséquences eu égard au développement de la traduction machine? Peut-on empêcher la mécanisation des pratiques traductives? Et inversement, est-il possible de cultiver l'indécidable au sein même de la traduction automatique? La démarche réflexive adoptée dans cette étude sonde la binarité qui sous-tend la théorie de Meschonnic pour tenter de penser la traduction par-delà l'opposition homme-machine, continu-discontinu, poétique-signe. Son objectif n'est pas tant d'apporter des solutions définitives à ces problèmes, mais bien plutôt d'interroger une partie des contradictions qui détermine notre rapport au traduire aujourd'hui. Par-delà son aspect performatif et ironique, qui s'inscrit dans une approche délibérément déconstructionniste, cet article soulève des questions d'ordre théorique, technique et éthique, qui méritent sans doute plus que jamais d'être étudiées relativement au recours accru à la traduction automatique, aussi bien par le grand public (Hutchins 2009: 16) que parmi les traducteurs professionnels (Commission européenne 2011) ${ }^{1}$. 


\section{Limites de la certitude}

En vue d'examiner la portée de la proposition de Meschonnic concernant l'absence de réflexion dans la traduction mécanique, je procède ici à une analyse comparée des traductions qu'en proposent trois outils de traduction automatique en ligne: Google Traduction, Babelfish et Dictionary.com. Voici tout d'abord le résultat pourvu par Google Traduction, le $1^{\text {er }}$ septembre 2014:

We can therefore consider that the major problem of translation is his (1) theory of language. That is, from the outset, involve (2) two things: the inseparability of what is known as a theory and a practice called (3), that is to say that practice is not a practice if it is not reflective or thoughtful, it is learned that ânonnement (4) of revenue (5) and if it is this reflexivity, this practice necessarily involves a comprehensive theory of language, and conversely a theory of translation would not (5) the reflection of a practice would only (6) the language of the (7) language applied to the (8) discourse, that is to say the (9) non-thinking ${ }^{2}$.

Cette traduction présente plusieurs problèmes sur les plans syntaxique et lexical, qui me serviront d'exemples pour analyser la nature de la réflexivité déployée au cours du processus décisionnel traductif. En (1), le déterminant possessif masculin his est proposé comme équivalent de l'adjectif possessif français sa au lieu du possessif neutre its qui accompagne les noms communs de genre neutre en anglais. En (2), l'agent grammatical est omis, de sorte que le verbe involve se trouve sans sujet. En (3), on assiste à l'inversion du sujet et du complément: ce que l'on appelle une pratique devient a practice called. En (4), Google ne parvient pas à traduire le terme ânonnement en anglais et décide de remployer le mot français tel quel. En (5), la traduction de recettes par revenue (au lieu de recipes) est un faux sens qui signale un «choix» inadéquat de la machine parmi les correspondances terminologiques disponibles. En (5) et (6), l'occurrence du verbe être au conditionnel est omise par le système de traduction automatique, qui ne réussit à en rendre que le mode (would). Enfin, en (7), (8) et (9), l'article défini the est employé à tort avec des noms abstraits tels que language, discourse et non-thinking.

Ces erreurs exposent les limites de la traduction automatique comme mécanisme fondé sur des correspondances terminologiques préétablies. Dans ce cas précis, la machine ne parvient à faire face ni aux ambiguïtés lexicales (la polysémie du mot recette), ni à la complexité syntaxique (l'emploi d'articles possessifs neutres), ni à la singularité idiomatique du texte de Meschonnic (l'occurrence du mot ânonnement). Ces fautes mettent ainsi en lumière les problèmes spécifiques d'une activité traductive qui «n'est pas réflexive ou réfléchie» (Meschonnic 2008: 59). À en juger par l'exemple qui suit, et malgré le secret industriel qui entoure le fonctionnement de Google Traduction, ce dernier n'est manifestement pas le seul outil de traduction automatique à opérer de cette façon. Le même jour en effet, le système Babelfish proposait une traduction de l'extrait de Meschonnic tout à fait identique à celle de Google Traduction:

We can therefore consider that the major problem of translation is his (1) theory of language. That is, from the outset, involve (2) two things: the inseparability of what is known as a theory and a practice called (3), that is to say that practice is not a practice if it is not reflective or thoughtful, it is learned that ânonnement (4) of revenue (5) and if it is this reflexivity, this practice necessarily involves a comprehensive theory of language, and conversely a theory of translation would not (5) the reflection of a prac- 
tice would only (+6) the language of the (7) language applied to the (8) discourse, that is to say the (9) non-thinking.

En plus de témoigner du fonctionnement similaire des deux outils, l'identité frappante des traductions pourvues par Babelfish et Google Traduction signale la difficulté de trouver aujourd'hui une pratique dite mécanique de la traduction qui ne comporte pas d'erreurs lexicales ou grammaticales, même basiques, puisque dans les deux cas les résultats obtenus sont incongrus (the non-thinking), incohérents (his theory of language), voire complètement incompréhensibles (it is learned that ânonnement of revenue).

Largement reconnue et discutée par les spécialistes (Arnold 2003; Hutchins 2009; Forcada 2010), la mauvaise qualité des traductions exécutées par les systèmes de traduction automatique s'observe par-delà la diversité des outils existants. L'exemple ci-dessous, tiré du système de traduction automatique Dictionary.com, montre en effet que l'incapacité de la machine à produire un texte intelligible et adéquat fait pour l'instant partie intégrante de ces systèmes, même si celle-ci peut se manifester de manière différente d'un outil à l'autre:

It (1) can therefore be assumed (2) that the major problem of the (3) translation is his (4) theory of language. This is good (5), from the outset, involve (6) two things: the inseparability between what is called a theory and what is called a practice, that is to say that a practice is not a practice if it is not reflexive or reflected, this is only a ânonnement of revenue learned (6), and if it is this reflexivity, this practice necessarily implies a theory of all of the (7) language; and conversely a theory of translation which would not be the reflection of a practice would (8) be that of language of the (8) language applied to the speech (9), that is to say the (10) non-thinking ${ }^{4}$.

Dans cet exemple, les mots sont soulignés lorsque le résultat proposé par Dictionary.com diffère de celui pourvu par Google Traduction et Babelfish. On note ainsi la substitution du pronom personnel we par it en (1); le recours à la voix passive là où Google Traduction et Babelfish emploient une forme active en (2); et le choix de traduire discours par speech plutôt que par discourse en (3). La police en gras signale quant à elle non seulement les erreurs grammaticales et lexicales qui, comme dans les traductions de Google ou Babelfish, obscurcissent la compréhensibilité du texte cible, mais aussi les éléments syntaxiques ou terminologiques qui opèrent une modification du texte source.

C'est le cas notamment du remplacement de la construction active On peut donc considérer que par la forme passive It can therefore be assumed; de la traduction de l'adverbe bien par l'adjectif good et du choix de traduire recette par revenue au lieu de recipe. Les traductions proposées par Dictionary.com dans ces exemples ne sont pas fausses en elles-mêmes : la formulation passive It can therefore be assumed est grammaticalement correcte; le terme bien peut en effet être traduit par good dans certains cas; et l'équivalent du mot recette dans un contexte économique serait effectivement revenue. C'est l'inadéquation des choix traductifs par rapport au texte source qui pose problème, c'est-à-dire l'inaptitude du système informatique à proposer un équivalent qui corresponde au sens et à la construction du texte de départ, son incapacité à tenir compte du contexte dans lequel l'énoncé apparaît. Comme semblent l'indiquer ces exemples, la réflexivité à l'œuvre dans l'acte traductif ne relève donc pas seulement de la capacité à produire un texte intelligible dans la culture cible, elle tient aussi à la faculté d'interpréter le texte source. L'obstacle majeur au bon fonctionnement de la 
traduction machine tient à l'ambigüité même du langage, au fait qu'il puisse exister plusieurs interprétations pour un seul texte et qu'une idée puisse être exprimée de multiples façons. De ce point de vue, la réflexivité en traduction consiste avant tout à pouvoir décider face à l'ambigu.

Si les limites de l'automation soulèvent des difficultés d'ordre linguistique, elles ne constituent pas selon Hatim et Mason (1990) des problèmes de traduction à proprement parler. Les défis posés par les systèmes de traduction mécanique n’appartiennent pas, d'après eux, au domaine traductif car ils ne procèdent pas d'une prise de décision véritable. C’est pourquoi, affirment-ils, malgré l'amélioration constante des outils à la disposition des traducteurs humains, la tâche principale de ces derniers reste la même: décider (Hatim et Mason 1990: 23). Hatim et Mason décrivent la réflexion déployée au cours du processus décisionnel traductif en ces termes:

1. Comprehension of source text:
a. parsing of text (grammar and lexis);
b. access to specialised knowledge;
c. access to intended meaning.

2. Transfer of meaning:

a. relaying lexical meaning;

b. relaying grammatical meaning;

c. relaying rhetorical meaning, including implied or inferrable meaning, for potential readers.

3. Assessment of target text:
a. readability;
b. conformity to generic and discoursal TL conventions;
c. judging adequacy of translation for specified purpose.

(Hatim et Mason 1990: 21)

Bien qu'intentionnellement simplifiée, cette liste des différentes facultés auxquelles fait appel une tâche traductive résume bien, me semble-t-il, l'étendue du processus décisionnel à l'œuvre dans la traduction humaine, depuis la compréhension du texte source jusqu'à l'évaluation du texte cible.

Contrairement à la machine, la traduction humaine cherche à prévenir les erreurs grammaticales et les différences sémantiques qui pourraient survenir au cours de l'acte traductif. Il est peu probable, par exemple, qu'un traducteur qualifié ou expérimenté emploie un article défini avec des noms abstraits en anglais ou qu'il traduise recette par revenue dans le contexte employé par Meschonnic. Une traduction humaine, comme celle que je propose ci-dessous, s'efforcera au contraire de déjouer les possibles erreurs lexicales ou syntaxiques afin d'assurer la compréhensibilité du texte cible et son adéquation au texte source:

We can therefore consider that the main problem of translation is its theory of language. From the outset, this implies two things: the inseparability of what is known as theory and what is called practice, that is to say that a practice is not a practice if it is not reflective or thoughtful, it is just a hesitant repetition of pre-existing codes [un ânonnement de recettes apprises]; but if it is reflective, such practice necessarily involves a comprehensive theory of language; and conversely, a translation theory that is not also a reflection on a particular practice would just be linguistics applied to discourse, that is to say non-thinking. (Meschonnic 2008: 60; notre traduction)

La réflexivité à l'œuvre dans la traduction humaine va au-delà de la capacité à enrayer les erreurs linguistiques. Elle relève de la prise de décision véritable, au sens 
derridien, c'est-à-dire de l'aptitude à choisir par-delà des propositions existantes, par-delà des correspondances préétablies. C'est le cas notamment de l'expression ânonnement de recettes apprises qui ne trouve pas de correspondance directe en anglais. Ne trouvant pas, comme Google Traduction, Babelfish et Dictionary.com, de terme anglais équivalent au mot ânonnement dans les dictionnaires bilingues consultés ${ }^{5}$, j'ai choisi contrairement à eux de proposer une traduction alternative - celle qui me semble le mieux évoquer l'idée d'ânonnement de recettes apprises dans ce contexte, à savoir a hesitant repetition of pre-existing codes. Dans ce cadre, ôter la réflexivité du processus traductif revient donc à dépouiller la traduction de sa faculté de choisir en l'absence de choix, c'est-à-dire à lui soustraire sa capacité à décider devant l'incertain.

\section{Au-delà des certitudes}

En traductologie, l'opposition entre le mécanique et l'humain repose principalement sur l'idée que, puisque l'automation opère selon des lois fixes et des correspondances terminologiques préétablies, la traduction machine ne requiert pas de prise de décision. Or, opposer la traduction automatique et la traduction humaine en ces termes ne rend pas compte du fait que les équivalences qui rendent le fonctionnement de la machine possible sont elles-mêmes le produit d'une décision humaine. Il existe actuellement trois types de traduction automatique: les systèmes à base de règles («Rule-based» MT), les systèmes de traduction automatique statistique (SMT), et les systèmes hybrides qui, comme leur nom l'indique, combinent le fonctionnement des systèmes à base de règles et des systèmes statistiques (Kenny et Doherty 2014: 276). Les logiciels de traduction automatique à base de règles créent la traduction à l'aide de dictionnaires intégrés et d'un ensemble de règles linguistiques applicables à la paire de langues concernée; les logiciels de traduction automatique statistique traduisent quant à eux en utilisant des modèles construits à partir d'un ensemble de textes monolingues et bilingues; les systèmes hybrides, enfin, conjuguent ces deux modèles (Koehn 2010: 314). Dans chaque cas, le fonctionnement de la machine s'appuie donc sur une prise de décision humaine: dans le cas de la traduction automatique à base de règles, les correspondances terminologiques et les calculs linguistiques sont établis par des linguistes, des terminologues et des informaticiens; dans les logiciels de traduction automatique statistique, la computation est basée sur des traductions humaines réelles; et au sein des modèles hybrides, la machine allie la réflexion des linguistes aux décisions concrètes des traducteurs (Forcada 2010: 220).

L'intervention humaine est omniprésente à tous les stades du fonctionnement de la traduction machine, à commencer par le choix du texte à traduire jusqu'au travail de post-édition des résultats pourvus par l'ordinateur. Inversement, la plupart des outils de traduction automatique reposent sur un processus décisionnel analogue au système cognitif humain (sélection de termes en fonction d'une combinaison complexe de paramètres sémantiques, grammaticaux et contextuels). Notons par ailleurs que les systèmes de traduction automatique statistique, comme Google Traduction, traduisent à partir de traductions humaines réelles: ils possèdent en ce sens une certaine faculté d'apprentissage (Kenny et Doherty 2014: 278). Ainsi, alors qu'en septembre 2014 Google Traduction ne proposait pas d'équivalent anglais pour le mot ânonnement et traduisait recette par revenue, une recherche plus récente montre que, 
depuis ma dernière consultation, la machine a "appris» la signification du terme ânonnement (droning) et qu'elle est désormais capable de choisir la traduction adéquate pour le mot recette (recipe) :

We can therefore consider that the major problem of translation is his theory of language. What is good from the outset involve two things: the inseparability between what is called a theory and a practice called, that is to say that a practice is not practice if it is not reflexive or reflected, this is just droning of recipes learned and if this reflexivity, this practice necessarily imply a general theory of language; and conversely a theory of translation that is not the reflection of a practice would be only linguistic language applied to the speech, that is to say, non-thought ${ }^{6}$.

Même si le résultat pourvu par la machine reste maladroit (droning of recipes), cet exemple confirme la capacité d'évolution des outils de traduction automatique et souligne le rôle primordial qu'y jouent les traducteurs humains puisque, comme le rappellent Way et Hearne (2011: 238), ce sont eux qui fournissent tout le savoir sur lequel les modèles de traduction automatique sont basés. Le fait que l'intervention humaine est indispensable au fonctionnement de la traduction machine, et que l'interaction entre traduction automatique et traduction humaine est si étroite, laisse supposer que la traduction machine n'est peut-être pas aussi irréfléchie qu'elle ne le semble de prime abord - ou plutôt, que ce qu'il y a d'irréfléchi et de mécanique en elle ne doit pas être opposé à la traduction humaine mais compris comme en dérivant.

L'argument selon lequel la traduction est un processus intrinsèquement réflexif présume qu'une traduction humaine est forcément le produit d'une décision réfléchie. Or en tant qu'opération linguistique, la traduction ne peut entièrement échapper à la mécanicité du langage. Car c'est justement la codification des unités linguistiques qui rend la traduction possible. Si, comme Meschonnic, Derrida remet en cause la distinction signifiant/signifié (en montrant notamment que tout signifié occupe aussi une position de signifiant ${ }^{7}$ ), contrairement à Meschonnic, il insiste également sur la nécessité de maintenir cette différence sans laquelle aucune pratique de la traduction ne serait possible:

il ne s'agit pas [souligne-t-il] de confondre à tous les niveaux et tout simplement, le signifiant et le signifié. Que cette opposition ou cette différence ne puisse être radicale et absolue, cela ne l'empêche pas de fonctionner et même d'être indispensable dans certaines limites - de très larges limites. Par exemple, aucune traduction ne serait possible sans elle. (Derrida 1968: 138)

Pour Derrida, la traduction est inhérente au concept même de signe, puisque dans un rapport de sémiosis le signe renvoie toujours à un autre signe, qui fonctionne ainsi comme son signifié, comme son interprétant. Ce qui établit le signe comme code dans la pensée derridienne, c'est sa répétabilité, son itérabilité (Derrida 1971: 72). L'itérabilité est la condition structurelle de l'avènement du signe, précise Derrida, puisqu'un signe ne pourrait se constituer comme tel (c'est-à-dire comme code) sans cette aptitude à être répété (Derrida 1971: 72). Sans répétabilité, la traduction ne pourrait pas exister. Par ailleurs, comme le souligne à juste titre David Bellos (2011: 266), une grande partie du travail des traducteurs professionnels repose sur la codification et la répétition. Les traducteurs expérimentés, explique-t-il, font souvent appel à des phrases types, à des formulations standard ou à des expressions courantes lorsqu'ils traduisent. Ils savent qu'il existe de nombreux mécanismes de transposition 
dans leurs langues de travail -que le pronom impersonnel «on» par exemple nécessitera presque toujours une voix passive en anglais. Ils développent du reste souvent leurs propres automatismes à mesure qu'ils acquièrent de l'expérience (mécanismes auxquels ils ont plus ou moins recours en fonction de leur familiarité avec le texte à traduire, du temps alloué, du gain perçu, etc.). En ce sens, ils se comportent comme Google Traduction, scannant leurs propres «mémoires» à la recherche de solutions traductives (Bellos 2011: 266).

Tout comme la traduction automatique, la traduction humaine s'appuie sur la mécanicité du langage, sur la répétabilité du signe. Or, du fait de cette itérabilité, tout signe dépasse du même coup la codification dont il procède. L'occurrence d'un signe dans un énoncé scelle son double statut en tant que code qui, en raison de sa possible transposition dans un autre contexte, excède la codification qui rend sa recontexualisation possible. "Il y a de la machine partout, et notamment dans le langage», note Derrida. «Or il existe dans la machine un excès par rapport à la machine elle-même [...] quelque chose qui déjoue le calcul machinal» (Derrida 2001: 87). Ce qui « excède la machine», pour Derrida, c'est l'indécidable - ce domaine qui «n'appartien[t] plus à l'ordre du calcul», «l'évènement qui par essence [reste] imprévisible et donc non

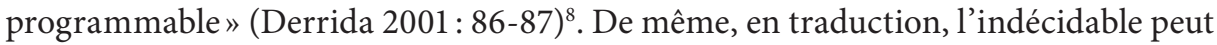
survenir partout, dans la machine comme dans l'humain. Le mot ânonnement en est un bon exemple. Sa non-traduction par Google Traduction signale à la fois la mécanicité de l'outil informatique (sa programmabilité) et cet excès de la machine par rapport à elle-même (le fait que tout ne peut être calculé, que l'incalculable est partout, y compris dans la machine). D'autre part, la capacité de l'outil à traduire un mot dont il ne connaissait pas le sens quelques mois auparavant montre bien que chaque nouvelle association de mots, chaque combinaison linguistique est elle-même fondée sur une prise de décision, sur ce qui - à un moment donné - résistait au mécanique et au programmable. De la même manière, dans ma traduction du commentaire de Meschonnic, l'intraduisibilité du terme ânonnement (le fait qu'il n'existe pas d'unité lexicale correspondant à ce mot dans les dictionnaires français-anglais consultés) m'oblige à faire face à l'incalculable, puisque je ne puis me contenter de choisir parmi des traductions existantes; je dois créer ma propre alternative, ma propre correspondance terminologique. Rompant et soulignant à la fois la codification à l'œuvre dans l'acte traductif, le terme ânonnement me force à générer un nouveau code, à produire une nouvelle association de mots, à le faire correspondre avec repetition. C'est en ce sens que traduire implique une prise de décision. Les traducteurs doivent prendre des décisions car il leur faut réagir devant l'incalculable, car il leur faut créer face à l'inconnu, car il leur faut répondre d'un choix qui excède toute prévision.

Pour Derrida, «sans l'épreuve [...] de l'incalculable, aucune décision responsable ne pourrait advenir. Décider, c'est prendre une responsabilité éthique et politique» (Derrida 1990 : 210). Une décision n'est donc responsable et éthique que lorsqu'elle excède le domaine du calculable et du mécanique:

L'épreuve du peut-être à laquelle soumet l'indécidable, c'est-à-dire la condition de la décision, ce n'est pas un moment qu'on excède, oublie, anéantit. Elle continue de constituer la décision comme telle, elle ne se sépare plus jamais d'elle, elle la produit comme décision dans et à travers l'indécidable, il n'y en a pas d'autre... l'instant de la décision doit rester hétérogène à tout savoir en tant que tel, à toute détermination théorique ou constative, même si elle peut et doit être précédée par toute la science et la conscience 
possibles. Ces dernières ne peuvent déterminer le saut de la décision sans transformer celle-ci en application irresponsable d'un programme, donc sans la priver de ce qui fait d'elle une décision souveraine et libre, une décision en un mot - s'il y en a jamais. (Derrida 1993: 247)

De ce point de vue, la traduction responsable et éthique serait celle qui dépasse la mécanicité du langage, celle qui est tournée vers l'inconnu. Une traduction responsable ne consisterait donc pas uniquement à "prendre» des décisions (choisir parmi plusieurs possibilités), mais nécessiterait de les «faire» (d'en créer de nouvelles, de former des correspondances inattendues, inconnues) - comme le suggère le gérondif making dans la locution anglaise decision-making. C'est d'ailleurs dans cette optique que Meschonnic parle de «poétique» du traduire, dans l'ouvrage du même titre. Pour Meschonnic (1999: 11-12), le processus traductif est un acte poétique au sens fort. C'est un acte créateur, une démarche transformatrice, une activité poétique dans l'acception étymologique du terme - du grec poieisis, «faire».

Le potentiel créatif de l'acte traductif est présent dans la traduction machine comme dans la traduction humaine car la signification de tout texte est indécidable, soumise à l'incertitude, ouverte à l'inconnu, qu'on le traduise automatiquement ou non. Comme le fait remarquer Kathleen Davis, « [t]he meaning of any text is undecidable, since it is an effect of language and not something that can be extracted and reconstituted. Translators must therefore make decisions in this strong sense» (Davis 2001 : 51). L'intraduisibilité du mot ânonnement est une manifestation extrême de cette indécidabilité, qui nous rappelle que même lorsque les mots sont traduisibles (c'est-à-dire lorsque des correspondances sont déjà établies dans une combinaison de langues donnée), une décision reste néanmoins à «faire», puisque la réutilisation même de cette association, sa répétition dans un contexte différent, peut créer autre chose, quelque chose de nouveau. En ce sens, avoir recours à la traduction automatique est en soi l'expression d'une décision, dont les traducteurs doivent être tenus pour responsables - la question n'étant plus dès lors de démêler l'automatique de l'humain, mais d'ouvrir le mécanique et le programmable à l'incertain, indépendamment d'où ils se manifestent, dans la traduction machine comme dans la traduction humaine. Car, comme le rappelle Derrida, «[s]avoir "prendre en compte” ce qui défie le compte à rendre [...] ne pas dénier ou ignorer cette venue imprévisible et incalculable de l'autre, c'est aussi cela le savoir, et la responsabilité scientifique» (Derrida 1990: 210).

\section{Vers plus d'incertitude}

Le rapport entre le machinal et le non-machinal est plus complexe qu'un rapport de simple opposition entre mécanique et humain. La machine est partout, dans les programmes informatiques comme dans le langage humain; mais tout langage, aussi mécanique soit-il, déborde l'ordre du programmable et du calculable qui permet son existence. Tout langage est hanté par l'irruption potentielle de l'imprévisible. Toute décision linguistique est traversée par «l'épreuve du peut-être à laquelle soumet l'indécidable» (Derrida 1993: 247). C'est pourquoi, soutient Derrida, il faudrait tenter de penser l'indécidable «avec la machine», et non contre elle (Derrida 2001 : 87). Le problème majeur des outils de traduction automatique statistique aujourd'hui ne réside pas tellement dans leur fonctionnement mécanique, mais dans la dissimulation du caractère incertain du résultat pourvu par l'ordinateur, c'est-à-dire dans la non- 
reconnaissance de cette part d'indécidabilité qui peut et doit survenir dans toute décision traductive responsable. Les outils de traduction automatique statistique comme Google Traduction font croire, par leur simplicité d'utilisation, que l'acte traductif repose sur un fonctionnement purement automatique, sur un mécanisme capable d'opérer sans sujet (Cronin 2012: 47). Or ils dissimulent par là tout le travail humain qui produit les données sur lesquelles ils se basent; ils font oublier les milliers d'informaticiens qui construisent ces systèmes en amont; ils donnent l'impression que la participation de l'utilisateur est superflue, voire inutile (Kenny et Doherty 2014: 288). Dans la plupart des systèmes de traduction automatique disponibles gratuitement en ligne aujourd'hui, tout se passe comme si aucune prise de décision n'était requise, comme si la traduction pouvait n'être que purement automatique, comme si elle ne relevait d'aucune responsabilité humaine.

La majorité des outils actuels n'incitent pas les utilisateurs à remettre en question la valeur des résultats pourvus par la machine. Le manque de transparence de leur fonctionnement et l'apparente univocité des traductions qu'ils proposent n'encouragent pas les usagers les moins avertis à adopter une attitude critique relativement aux résultats obtenus. Or, une foi absolue en l'outil peut se révéler problématique car elle peut non seulement empêcher une prise de décision véritable, et déresponsabiliser l'utilisateur, mais aussi conduire à des malentendus et nier la visée communicationnelle de l'acte traductif. À titre d'exemple, je souhaiterais revenir sur une petite anecdote personnelle. J'étais il y a quelques mois en déplacement pour une conférence à Reykjavik. J'avais réservé mon logement en ligne - une chambre d'hôte chez une dame islandaise qui ne parlait ni français ni anglais. Ne parlant pas moi-même l'islandais, nous avons communiqué tant bien que mal pendant mon séjour, limitant nos échanges à des salutations polies et des hochements de tête. De retour en Angleterre, je laissai un commentaire sur sa page personnelle et en échange je trouvai le message suivant: "Very good guest, comfortable and used widely finished fluoroscopically.». Le premier tiers de la phrase est clair et laisse présager un commentaire globalement positif. Le second est légèrement plus surprenant, puisque comfortable se dit d'un objet et non pas d'une personne. Je commence rapidement à soupçonner le recours à un outil de traduction automatique - soupçon qui se trouve accentué par l'incompréhensibilité totale du troisième tiers de la phrase (used widely finished fluoroscopically). De toute évidence, ce commentaire n'est pas l'œuvre d'un traducteur expérimenté. Ce n'est pas non plus le produit d'une traduction littérale à l'aide d'un dictionnaire bilingue où l'usage des mots est contextualisé. Si elle avait utilisé un dictionnaire, mon hôtesse aurait vu par exemple que la signification du mot islandais rólegur change selon les contextes, qu'il peut se traduire par comfortable ou par calm. Le fait que mon hôtesse ait décidé de publier un commentaire dont elle ignore manifestement le sens suggère au contraire une confiance absolue en l'outil de traduction utilisé, auquel elle semble se remettre assurément et sans questionnement.

$\mathrm{Au}$ lieu de faciliter une mise en relation avec l'autre, une foi intégrale en la traduction machine peut au contraire entraver les échanges, tout en donnant à l'émetteur l'illusion d'une communication réussie. Dans le cas décrit, l'enjeu est bénin, mais dans d'autres circonstances les conséquences peuvent se révéler plus graves. Lors du tournage de mon film documentaire sur le service de traduction du Programme des Nations Unies pour le développement (PNUD) en avril 2014, j'ai assisté à une utilisation quelque peu problématique de la traduction automatique dans un cadre 
institutionnel. Contrairement aux autres agences de l'ONU, le PNUD fait traduire la majorité de ses textes par des traducteurs externes sous la direction de son Bureau de la communication. Ainsi, lorsqu'un communiqué de presse doit être traduit dans l'une des langues officielles, le responsable du service fait appel à un traducteur professionnel externe. Ce fut notamment le cas d'un communiqué de presse consacré aux stratégies du PNUD pour la jeunesse. Or, parallèlement à la traduction réalisée par le traducteur professionnel, ce communiqué fut également traduit par un employé interne à l'aide d'un système de traduction automatique en ligne. Et c'est cette version automatique, dont la qualité laissait grandement à désirer, qui fut publiée sur le site internet de l'organisation. Lorsque le responsable du service de traduction découvrit la publication de cette version, il reconnut aussitôt les tournures de phrase bancales de Google Traduction et demanda que l'on remplace la version automatique par la traduction professionnelle. L'employé en question, qui n'était pas lui-même traducteur, ne semblait pas conscient des enjeux d'une telle démarche. Or, les répercussions peuvent être critiques: non seulement parce qu'une traduction défectueuse peut ternir l'image de l'organisation et donc dissuader les pays donateurs de continuer à contribuer à son fonctionnement, mais aussi car elle peut susciter des malentendus diplomatiques, voire générer des tensions politiques.

On le voit bien, ce n'est pas la traduction machine en tant que telle qui pose problème mais l'utilisation purement mécanique qui en est faite par l'usager, c'est-àdire la déresponsabilisation de ce dernier relativement au produit proposé par l'ordinateur. Ce que montrent ces exemples, c'est que dans toute traduction automatique la prise de décision ne peut advenir que par-delà les choix opérés par les programmes informatiques: elle ne peut survenir qu'au moment de la réponse de l'utilisateur à la traduction proposée par la machine; elle ne peut s'exprimer que comme prise de responsabilité par rapport au système automatique - c'est-à-dire qu'au-delà du produit présenté par l'outil. Un emploi responsable de la traduction automatique exige donc que l'on reconnaisse l'indécidable au cœur même du mécanique. Il impose que l'on abandonne nos certitudes relativement aux résultats proposés par la machine. Il requiert que l'on remette en question la mécanicité même de la traduction automatique. Explorer l'imprévisible, l'incertain et le poétique au sein même de l'automatique, c'est précisément ce qu'entreprend Barbara Godard dans sa série de traductions automatiques du poème d'Apollinaire «Les Fenêtres». Dans One Poem in Search of a Translator (2009), Godard a recours à des outils de traduction mécanique tels que Systran et Promt pour explorer sa vision du poème comme «machine», où la traduction joue un rôle majeur en tant que «multiplicateur» ou "générateur de nouveaux poèmes» (Godard 2009: 207; notre traduction). Montrant que la traduction machine fonctionne «avec les matériaux existants, ready-made de la langue [...] pour façonner de nouveaux énoncés», les traductions automatiques de Godard soulignent «le processus de recontextualisation continuel de tout projet traductif» et semblent indiquer que la traduction machine peut elle aussi être "créative» (Godard 2009: 208; notre traduction). En mettant simultanément en scène la mécanicité de l'objet poétique et le potentiel créatif de la traduction automatique, l'expérimentation de Godard expose le caractère doublement machinal et imprévisible de toute création, qu'elle soit mécanique ou humaine.

Reconnaître l'indécidable au cœur du mécanique ne revient donc pas, précisonsle, à nier ou à rejeter la mécanicité dans son intégralité. Si la décision est ce qui 
«devrait rester imprévisible et donc non programmable», pour pouvoir y accéder il faut aussi «prendre en compte la programmation, la machine, la répétition, le calcul» (Derrida 2001 : 87). Il ne s'agit donc pas de savoir s'il faut ou non utiliser les outils de traduction automatique, mais de se demander comment on peut encourager une utilisation responsable de ces outils. Si une traduction responsable exige que l'on tienne compte de la part d'incertitude à l'œuvre dans toute opération linguistique, comment inciter les utilisateurs à un usage plus responsable de la traduction automatique? Comment favoriser une attitude plus incertaine, réflexive et plurielle relativement aux traductions proposées par la machine? Comment cultiver l'incertitude au sein même du mécanique? Comment pousser les utilisateurs à remettre en question leur confiance, parfois aveugle, en l'outil? Autrement dit, comment créer un système de traduction automatique autocritique et réflexif? Les possibilités ne manquent pas. Un outil pourrait par exemple proposer plusieurs traductions d'un même texte ou bien souligner les mots qu'il ne parvient pas à traduire; il pourrait également contextualiser l'utilisation des termes recherchés et indiquer la probabilité de leur occurrence dans un contexte donné. Une machine qui montrerait ses propres limites et qui reconnaîtrait la part d'incertitude nécessaire à sa bonne utilisation ferait non seulement gagner du temps aux traducteurs professionnels en les aidant à identifier les problèmes de traduction particulièrement épineux, mais elle alerterait aussi les utilisateurs moins avertis (comme mon hôtesse islandaise) des échecs possibles de la communication. Elle encouragerait donc ainsi les usagers à cultiver l'esprit de vigilance indispensable à une pratique responsable de la traduction.

La responsabilisation des traducteurs professionnels passe également par l'éducation. Dans une industrie où le recours aux logiciels de traduction assistée par ordinateur est de plus en plus répandu (Kenney et Doherty 2014: 276), la formation des traducteurs aux technologies de la traduction est sans doute plus importante que jamais. L'évaluation humaine demeure en effet indispensable à une utilisation responsable de la traduction automatique (White 2003: 244). Les traducteurs doivent acquérir les compétences de post-édition nécessaires pour mener à bien leur tâche. Dans certains cas, ce travail d'évaluation peut aussi se faire en amont, au moment de la préparation du texte à insérer dans l'outil, c'est-à-dire au stade de pré-édition du texte source (Hutchins et Somers 1992: 151). D’ailleurs, même lorsque les outils proposent des systèmes d'évaluation automatique intégrée, les traducteurs doivent être capables de comprendre le fonctionnement et les limites de ces métriques (Kenney et Doherty 2014: 285). De plus, s'il est vrai que les systèmes de traduction automatique en ligne comme Google Traduction sont à priori faciles d'accès et d'utilisation (Robinson 2012: 39), ils ne sont pas forcément destinés à un usage professionnel (EMT Expert Group 2009) ${ }^{9}$. Selon Kenney et Doherty (2014: 288), certaines clauses de confidentialité interdisent par exemple aux traducteurs de télécharger des documents sur la plateforme de traduction en ligne de Google, de sorte que dans la majorité des cas les traducteurs sont tenus d'utiliser l'outil que de façon ponctuelle et uniquement pour traduire des segments courts. De manière générale, utiliser les outils de traduction automatique en ligne à des fins professionnelles soulève des problèmes d'ordre juridique et éthique auxquels il faut sensibiliser les traducteurs à travers une formation approfondie et adaptée. 


\section{Conclusion}

Une traductologie opposant l'homme et la machine se révèle difficilement tenable en pratique car mécanicité et réflexivité font toutes deux partie intégrante du langage humain, dont la machine n'est elle-même qu'une extension. Tout comme la traduction humaine, la traduction automatique possède une part d'incertitude que les utilisateurs doivent reconnaître pour en assurer une utilisation éthique. Une traduction automatique ne peut devenir réflexive et responsable qu'en réalisant toute l'étendue de sa mécanicité, c'est-à-dire qu'en assumant la transgression du code qu'elle instaure, qu'en admettant la part d'indécidabilité qui règne au cœur même de sa programmabilité. Une pratique réflexive de la traduction automatique nécessite donc une reconnaissance de ses propres limites en tant qu'opération linguistique: elle exige que l'on fasse, à travers elle, l'expérience de l'impossible certitude du langage, que l'on franchisse l'épreuve de cet incalculable sans lequel aucune décision responsable ne pourrait advenir. Car si ôter la réflexivité du processus traductif signifie dépouiller la traduction de sa faculté de décider devant l'indécidable, une pratique éthique de la traduction doit à l'inverse faire ressortir le réflexif de l'automatique, cultiver l'incalculable dans le calculable, faire prospérer l'incertitude au cœur même de la certitude.

L'usage de la traduction automatique s'étend aujourd'hui à des domaines aussi variés que les institutions politiques, les réseaux sociaux ou le tourisme. Or les usagers ne sont pas tous conscients des limites de ces outils, de leurs lacunes, des imperfections qui rendent l'intervention humaine indispensable à tous les stades de leur utilisation. Grand nombre d'utilisateurs ignorent les incertitudes qui régissent le fonctionnement des outils de traduction automatique en ligne; ils négligent le fait que, sous couvert de certitude et de mécanicité, ces outils sont en mutation constante, qu'ils dépendent de décisions humaines réelles, qu'ils requièrent une évaluation attentive et vigilante. Il appartient donc aux systèmes de traduction automatique d'intégrer l'incertain au sein même de leur fonctionnement et d'encourager les utilisateurs à un usage averti et responsable de leurs outils. Ces systèmes se doivent d'être plus transparents à l'égard de leurs propres limites pour pousser les usagers à interroger la validité des traductions proposées et favoriser une participation humaine active et une attitude prudente relativement à l'outil.

Inversement, il est primordial que la traductologie s'intéresse à la machine et l'intègre dans ses réflexions car la traduction est continuellement définie par les technologies qu'elle utilise (Cronin 2003 ; 2012). On ne traduit pas de la même façon qu'il y a trente ans. Les nouvelles technologies ne cessent de transformer les pratiques traductives; il faut donc adapter les concepts et l'enseignement de la traduction pour rendre compte de ces nouvelles pratiques et y faire face (Pym 2013 : 487). Il est impératif en effet de responsabiliser les traducteurs professionnels à l'usage des systèmes de traduction automatique, de les former à comprendre le fonctionnement complexe des outils qu'ils peuvent être amenés à utiliser et de les aider à reconnaître la part d'incertitude qui sous-tend ces systèmes et exige leur participation active. Il faut, en d'autres termes, s'efforcer de sensibiliser les traducteurs aux zones d'indécidabilité que traverse toute démarche traductive responsable, qu'elle s'effectue ou non à l'aide d'un outil de traduction automatique. 


\section{NOTES}

1. Commission européenne (2011): Conclusions: Translator Profile. What skills for a changing market? Direction générale de la traduction de la CE, Consulté le 20 janvier 2015, <http://ec. europa.eu/dgs/translation/programmes/languageindustry/platform/documents/translator_profile _conclusions_en.pdf $>$.

2. Google Traduction: Consulté le $1^{\text {er }}$ septembre 2014, <https://translate.google.com/?hl=fr $>$.

3. Babelfish: Consulté le $1^{\text {er }}$ septembre 2014, <www.babelfish.com/>.

4. Dictionary.com, Consulté le $1^{\text {er }}$ septembre 2014, <http://dictionary.reference.com/>.

5. Collins French Dictionary: Consulté le $1^{\text {er }}$ septembre 2014, <www.collinsdictionary.com/dictionary/ english-french>.

6. Google Traduction, Consulté le 20 janvier 2015, <https://translate.google.com/?hl=fr >.

7. L'exemple donné par Geoffrey Bennington est celui du dictionnaire: «Cherchez dans le dictionnaire un signifié d'un signifiant inconnu, vous trouverez d'autres signifiants, jamais de signifiés» (Bennington et Derrida 1981: 36).

8. Ces citations sont extraites du quatrième chapitre de De quoi demain... dialogue avec Élisabeth Roudinesco (Derrida 2001). Répondant à une question concernant le scientisme contemporain qui, d'après Roudinesco, "va de pair avec la transformation de l'humain en machine» (2001: 83), Derrida examine le problème de la machine au sens large. Qu'elle soit réelle ou virtuelle, informatique ou psychique, technique, économique ou formelle, la machine désigne chez Derrida «un dispositif de calcul et de répétition» (2001: 86). Dans ce passage, Derrida insiste particulièrement sur l'omniprésence de la machine, sur le fait qu'elle se manifeste partout, qu'elle traverse tous les domaines. «Dès qu'il y a du calcul, de la calculabilité et de la répétition, il y a de la machine» (2001: 86), souligne-t-il. Or, et c'est là son argument central, la machine, où qu'elle se manifeste (dans l'inconscient, dans le langage ou dans la technique), comporte quelque chose d'incalculable, d'imprévisible. Je m'appuie ici sur l'articulation complexe des concepts de «machine» et d' 'indécidable» dans la pensée derridienne, pour tenter d'examiner le rapport biologique-mécanique en traduction au-delà d'une relation de simple opposition. Car, comme le rappellent Daniel Bougnoux et Bernard Stiegler, c'est «Jacques Derrida qui, dans De la grammatologie, mais aussi dans "Freud et la scène de l'écriture", a posé que l'écriture, c'est la technique. Tout ce qu'il dit de l'écriture est vrai de la technique en règle générale. C'est Derrida qui a montré que la technique permet la déterritorialisation, le contrôle, la standardisation, etc., et en même temps la singularité» (Bougnoux et Stiegler 2014: 89).

9. EMT Expert Group (2009): Competences for professional translators, experts in multilingual and multimedia communication. Bruxelles: EMT Expert Group. Consulté le 18 mars 2015, <http:// ec.europa.eu/dgs/translation/programmes/emt/key_documents/emt_competences_translators_ en.pdf $>$.

\section{RÉFÉRENCES}

Apter, Emily (2013): Against World Literature: On the Politics of Untranslatability. New York: Verso.

Arnold, Doug (2003): Why translation is difficult for computers. In: Harold Somers. Computers and Translation: A translator's guide. Amsterdam/Philadelphie: John Benjamins, 119-142.

Bellos, David (2011): Is That A Fish in your Ear? Londres: Penguin Books.

Benjamin, Walter (1923/2000): La tâche du traducteur. In: Euvres I (Traduit par Maurice DE Gandillac, Pierre Rusch et Rainer Rochlitz). Paris: Gallimard, 245-262.

Bennington, Geoffrey et Derrida, Jacques (1981): Jacques Derrida. Paris: Éditions du Seuil. Berman, Antoine (1984): L'épreuve de l'étranger. Paris: Gallimard.

Berman, Antoine (1999): La traduction et la lettre, ou L'auberge du lointain. Paris: Seuil.

Bougnoux, Daniel et Stiegler, Bernard (2014): Pour Jacques Derrida. In: Jacques Derrida. Trace et archive, image et art. Bry-sur-Marne: INA Éditions, 77-101.

Caputo, John D. (1997): Deconstruction in a Nutshell: A Conversation with Jacques Derrida. New York: Fordham University Press.

Commission européenne (2011) : Conclusions: Translator Profile. What skills for a changing market? Direction générale de la traduction de la CE. Consulté le 20 janvier 2015, <http:// 
ec.europa.eu/dgs/translation/programmes/languageindustry/platform/documents/translator_profile_conclusions_en.pdf >.

Cronin, Michael (2003): Translation and Globalization. Londres: Routledge.

Cronin, Michael (2012): Translation in the Digital Age. Londres: Routledge.

DAvis, Kathleen (2001): Deconstruction and Translation. Manchester: St Jerome.

DERrida, Jacques (1968): Sémiologie et grammatologie. Information sur les sciences sociales. 7(3):135-148.

DerRIDA, Jacques (1971): Signature, événement, contexte. In: Association DES SOCIÉTÉS DE PHILOSOPHIE De LANGUe FRANÇAISE. La Communication: Actes $d u X V^{e}$ Congrès de l'Association des sociétés de philosophie de langue française. Montréal: Montmorency.

Derrida, Jacques (1985): Des Tours de Babel. In: Joseph F. Graham, dir. Difference in Translation. Ithaca/Londres: Cornell University Press, 209-248.

Derrida, Jacques (1990): Limited Inc. Paris: Galilée.

Derrida, Jacques (1993): Spectres de Marx. Paris: Galilée.

Derrida, Jacques (2001): De quoi demain... dialogue avec Élisabeth Roudinesco. Paris: Fayard/ Galilée.

Forcada, Mikel L. (2010): Machine Translation Today. In: Yves Gambier et Luc Van DoorsLAER, dir., Handbook of Translation Studies. vol. 1. Amsterdam/Philadelphie: John Benjamins, 215-223.

GodArd, Barbara (2009): Translating Apollinaire after bp Nichol. In: Loffredo, Eugenia et Perteghella, Manuela. One Poem in Search of a Translator: Rewriting 'Les Fenêtres' by Apollinaire. Oxford: Peter Lang, 207-224.

Hatim, Basil et Mason, Ian (1990): Discourse and the Translator. Londres: Longman.

Hutchins, John (2009): Multiple Uses of Machine Translation and Computerised Translation Tools. International Symposium on Data and Sense Mining, Machine Translation and Controlled Languages-ISMTCL. Consulté le 20 janvier 2015, <www.hutchinsweb.me.uk>.

Hutchins, John et Somers, Harold (1992): An Introduction to Machine Translation. Londres: Academic Press.

Kenny, Dorothy et Doherty, Stephen (2014): Statistical machine translation in the translation curriculum: overcoming obstacles and empowering translators. The Interpreter and Translator Trainer 8(2):276-294.

Koenn, Philipp (2010): Statistical Machine Translation. Cambridge: CUP.

Ladmiral, Jean-René (2014): Sourcier ou cibliste. Paris: Les Belles Lettres.

Meschonnic, Henri (1999): Poétique du Traduire. Paris: Verdier.

Meschonnic, Henri (2008): Traduire au xxi ${ }^{e}$ siècle. Quaderns: Revista de traducció. 15:55-62.

Pyм, Anthony (2010): Exploring Translation Theories. Londres et New York: Routledge.

Pyм, Anthony (2013): Translation skill-sets in a machine-translation age. Meta. 58(3) : 487-503.

Reiss, Katarina et Vermeer, Hans (1984/2014): The Foundation of a General Theory of Translation (Traduit par Christiane NoRD). New York: Routledge.

RoBinson, Doug (2012): Becoming a Translator. $6^{e}$ éd. Londres: Routledge.

Scotт, Clive (2012): Translating the Perception of Text. Cambridge: Cambridge University Press.

Shuttleworth, Mark et Cowie, Moira (1997): Dictionary of Translation Studies. Manchester: St. Jerome.

Tyмосzко, Maria (2013): Translation Theory. In: Carol A. Chapelle, dir. The Encyclopedia of Applied Linguistics. Oxford: Blackwell.

Venuti, Lawrence (1995): The Translator's Invisibility. New York: Routledge.

Way, Andy et Hearne, Mary (2011): On the Role of Translations in State-of-the-Art Statistical Machine Translation. Language and Linguistics Compass. 5(5):227-248.

White, John S. (2003): How to Evaluate Machine Translation. In: Harold Somers, dir. Computers and Translation. A translator's guide. Amsterdam/Philadelphie: John Benjamins, 211-244. 